\title{
Research on Error Analysis and Correction Technique of Atmospheric Refraction for InSAR Measurement with Distributed Satellites
}

\author{
Guojun Hu',2,3, Li Zhang2,3, Gang Li2,3, Hui Gong2,3, Jinchun Qin²,3 \\ ${ }^{1}$ Institute of Geospatial Information, Information Engineering University, Zhengzhou, China \\ ${ }^{2}$ State Key Laboratory of Geo-information Engineering, Xi'an, China \\ ${ }^{3} \mathrm{Xi}$ 'an Research Institute of Surveying and Mapping, Xi'an, China \\ Email: hgj_2000@163.com
}

How to cite this paper: Hu, G.J., Zhang, L., Li, G., Gong, H. and Qin, J.C. (2016) Research on Error Analysis and Correction Technique of Atmospheric Refraction for InSAR Measurement with Distributed Satellites. Journal of Computer and Communications, 4, 142-150.

http://dx.doi.org/10.4236/jcc.2016.415014

Received: November 8, 2016

Accepted: November 25, 2016

Published: November 28, 2016

\begin{abstract}
SAR interferometry with distributed satellites is a technique based on the exploitation of the interference pattern of two SAR images acquired synchronously. The interferogram contains geometric, atmospheric, topographic and land defomation. This paper focuses on atmospheric effects on SAR interferometry, which shows theoretically that the relationship among ionosphere TEC and troposphere parameters such as temperature, relative humitdity and pressure with respect to slant rang changes. An atmospheric correction method is given in the end.
\end{abstract}

\section{Keywords}

InSAR, Atmospheric Refraction, Troposphere Delay, Ionosphere Delay, Error Correction

\section{Introduction}

With the development and the maturity of SAR Interferometry technology, its accuracy is increasing largely and it is significant to study the factors which can lower the accuracy of SAR Interferometry technology [1] [2]. SAR interferometry system with distributed satellites is based on the exploitation of the interference pattern of two SAR images acquired synchronously, from which useful information is extracted. Atmospheric refraction is one of the main errors because that the temporal and spatial uncertainty of atmospheric change causes different delay of radar signal [3] [4] [5] [6]. The paper discusses the fundamental theory of electromagnetic wave signal's atmospheric propagation delay and the main model of Troposphere delay correction and Ionosphere delay 
correction, establishing the relationship between tropospheric parameters and the distance change. Then the magnitude that Ionosphere and Tropospheric parameters exert influence on the ranging accuracy is analysed. The method to inhibit or eliminate the influence is discussed in the end.

\section{Fundamental Theory and Model}

\subsection{Troposphere Delay}

According to the Fermat principle and Snell's law in the basic theory of light propagation, the optical range $\rho$ from satellite to ground point is formed as

$$
\rho=\int_{r_{0}}^{r_{1}} \frac{n}{\cos \theta} d r
$$

where $r_{0}$ is the center distance for a ground point (Figure 1), r1 is the center distance for the top of neutral atmosphere, $\mathrm{z}$ is the view zenith distance of Any point at signal propagation path, $\theta$ is the view height Angle of Any point at signal propagation path, and $\mathrm{n}$ is atmospheric refractivity [7] [8] [9].

Define $\mathrm{N}$ for the difference of atmospheric refractive index:

$$
N=(n-1) \times 10^{-6}
$$

In a spherically symmetric layered medium, according to Snell law, a formula is given by

$$
n r \sin z=n_{0} r_{0} \sin z_{0}=\text { const }
$$

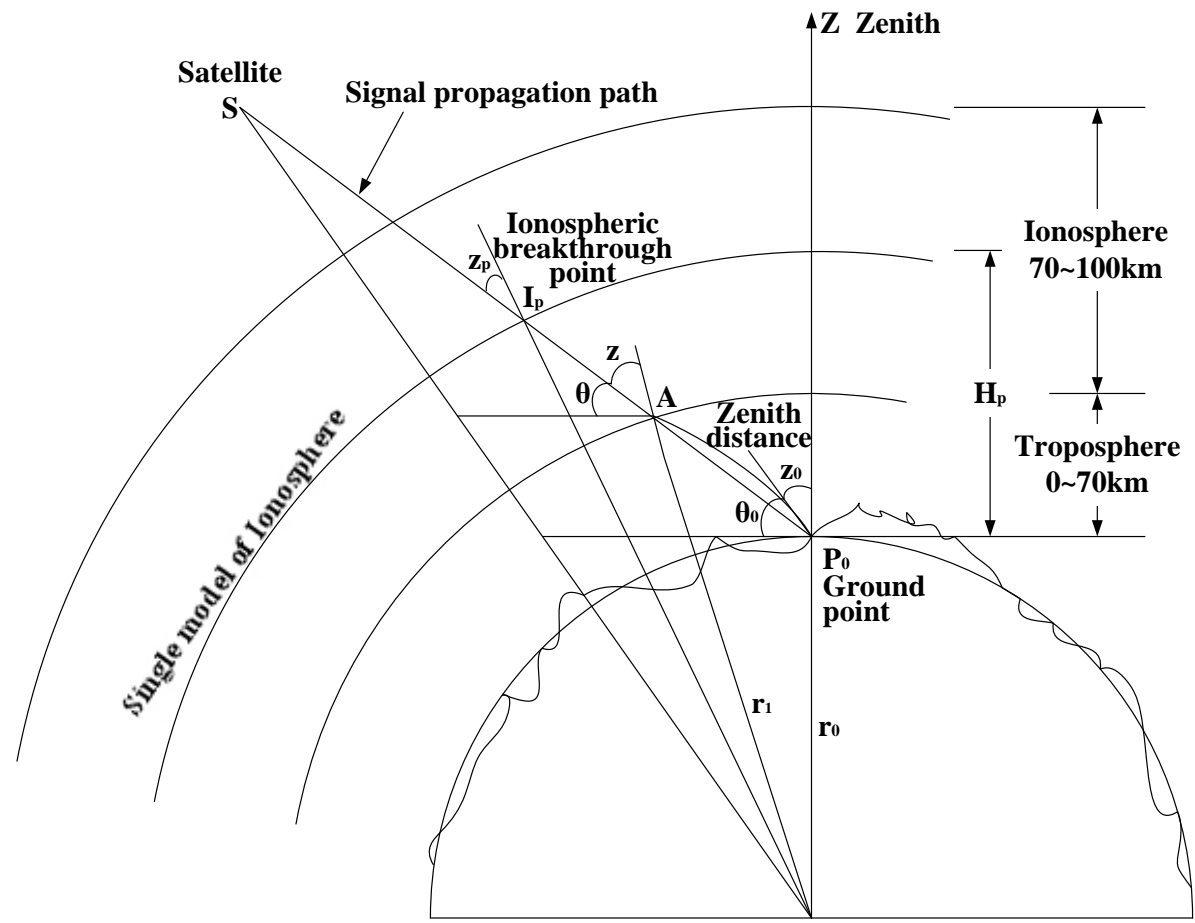

The earth's core $O$

Figure 1. Signal propagation path. 
$n_{0}, r_{0}, z_{0}$ are the corresponding values of ground measuring site. The path delay is given by the product of the tropospheric zenith path delay and the function of satellite-zenith distance $z$ (the so-called mapping function). Only considering the first order effect, mapping function $\mathrm{f}(\mathrm{z})$ is approximate to $1 / \cos z$.

$$
f(z) \approx f_{d}(z) \approx f_{w}(z) \approx \frac{1}{\cos z}
$$

According to Saastamoinen model (1972) of the atmospheric refraction, the zenith delay of atmospheric refraction can be given by

$$
\left.\begin{array}{l}
f(\Phi, H)=1-0.0026 \cos 2 \Phi-0.00028 H \\
\Delta \rho_{d}^{z}=0.002277 P / f(\Phi, H) \\
\Delta \rho_{w}^{z}=0.002277 e[0.05+1255 /(273.15+T)] / f(\Phi, H)
\end{array}\right\}
$$

where $\Delta \rho_{d}^{z}$ is the dry air delay of the zenith (dry item), $\Delta \rho_{w}^{z}$ is the water vapor delay of the zenith (wet item), $\mathrm{P}$ is the surface atmospheric pressure $(\mathrm{Pa}), \mathrm{H}$ is the atmospheric high $(\mathrm{km})$ of ground point, $\mathrm{T}$ is the surface temperature $\left({ }^{\circ} \mathrm{C}\right), \Phi$ is the geographic latitude of measuring station, $\mathrm{E}$ is the vapor pressure (hPa) [10] [11].

According to Magnus' empirical formula, the relationship between vapor pressure and relative humidity at the condition of air temperature $\mathrm{T}\left({ }^{\circ} \mathrm{C}\right)$ can be expressed as

$$
e=\frac{R_{h}}{100} \times 6.11 \times 10^{\frac{7.5 T}{237.3+T}}
$$

\subsection{Ionosphere Delay}

Due to variable insolation of the Sun the spatial distribution of the layers varies during the day. The impact of the state of the ionosphere on the propagation of waves is characterized by the Total Electron Content (E), where

$$
E=\int_{P_{0}}^{s} n_{e} d \rho
$$

The integral contains the total number of electrons that are included in a column with a cross-sectional area of $1 \mathrm{~m}^{2}$, counted along the signal path $\rho$ between the ground point $\mathrm{PO}$ and the satellite $\mathrm{S}$. The unit of measurement is the TECU (Total Electron Content Unit): 1 TECU $=1 \times 10^{16} \mathrm{el} / \mathrm{m}^{2}$.

The ionosphere is a dispersive medium for radio waves. For the formula of dispersion (e.g. Davies, 1990) of the index of refraction, rearranging and neglecting higher order terms gives [12] [13] [14]:

$$
n_{1}=1-\frac{a n_{e}}{f^{2}}
$$

where the parameter $\mathrm{a}$ is a constant.

According to Equations (7) and (8), the ionosphere delay along the signal propagation path $\Delta \rho^{I}$ is given by

$$
\begin{aligned}
& \Delta \rho^{I}=\int_{L}\left(n_{1}-1\right) d \rho=-\frac{a E}{f^{2}} \\
& a=40.3 \times 10^{16} \mathrm{~m} \cdot \mathrm{s}^{-2} \cdot T E C U^{-1}
\end{aligned}
$$


That is to say, if we can get the $\mathrm{E}(\mathrm{TEC})$ along the signal propagation path, we can calculate the propagation delay directly.

\section{Analysis of Influence on Ranging Accuracy Exerted by Atmospheric Refraction}

\subsection{Troposphere Atmospheric Refraction Error}

The atmospheric refractive effect of Troposphere is independent of the frequency but is decided by the atmospheric temperature, pressure and humidity. Atmospheric refractive error of Troposphere includes the dry item and the wet item. Refraction error of Tropospheric atmosphere in zenith direction is about $2.0-2.7 \mathrm{~m}$, which is computed approximately (Figure 2). Among them, the dry item of distance error caused by the refraction is about $2.4 \mathrm{~m}$ and the wet item is about $0.05-0.05 \mathrm{~m}$.

The inland distribution of distance error caused by Tropospheric atmosphere refraction, which is obtained by using the historical meteorological sounding data of 10 years, is shown in Figure 3.

From the above research on the inland distribution of distance error, we can get some conclusions as follow:

1) When the elevation of the satellite is above $30^{\circ}$ (viewed from the ground), the distance error caused by Tropospheric atmosphere refraction is mostly less than $5.5 \mathrm{~m}$.

2) The dry item of refractive error can be estimated accurately from the ground meteorological parameters due to its regular and steady changes. The wet item of refractive error is the main part of the residual error for refractive error correction due to its irregular changing with time and space.

3) The proportion changes of distance error caused by the wet item of the refraction vary with month and quadrillage obviously but do not vary with elevation.

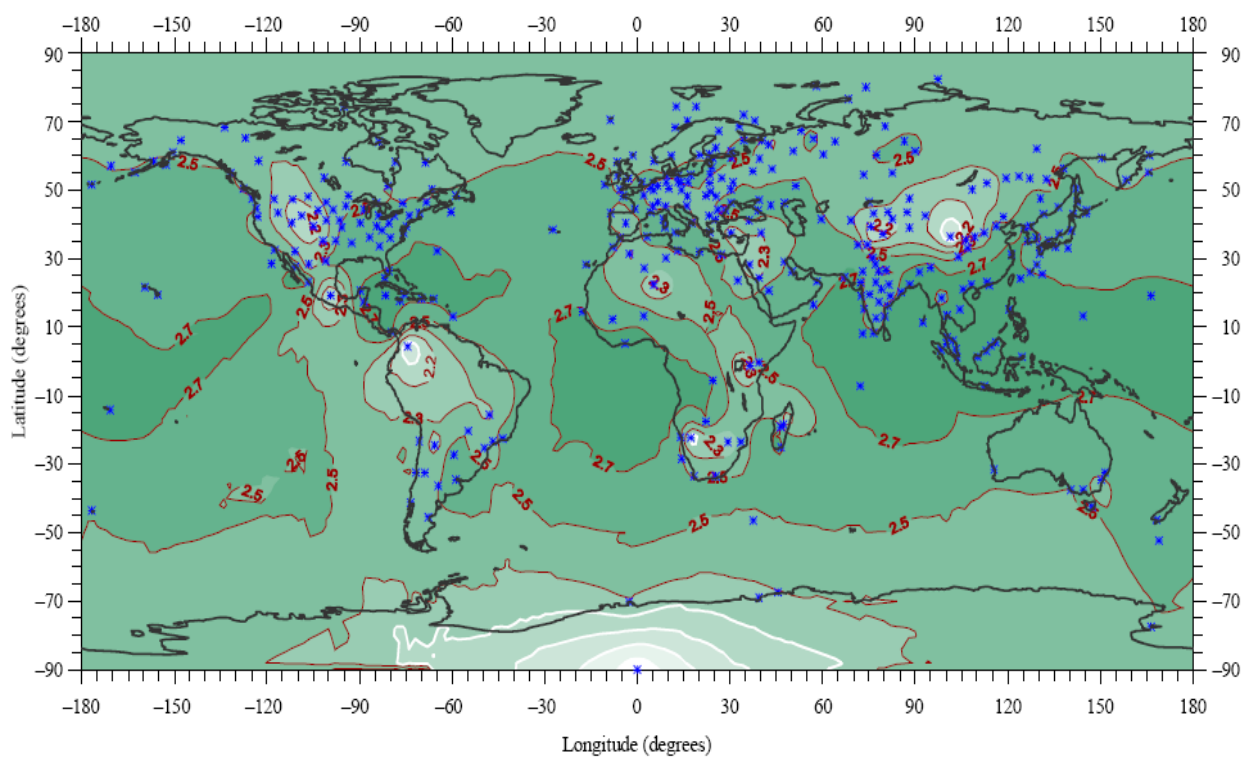

Figure 2. The global distribution of refraction error from ITU-R P.834. 

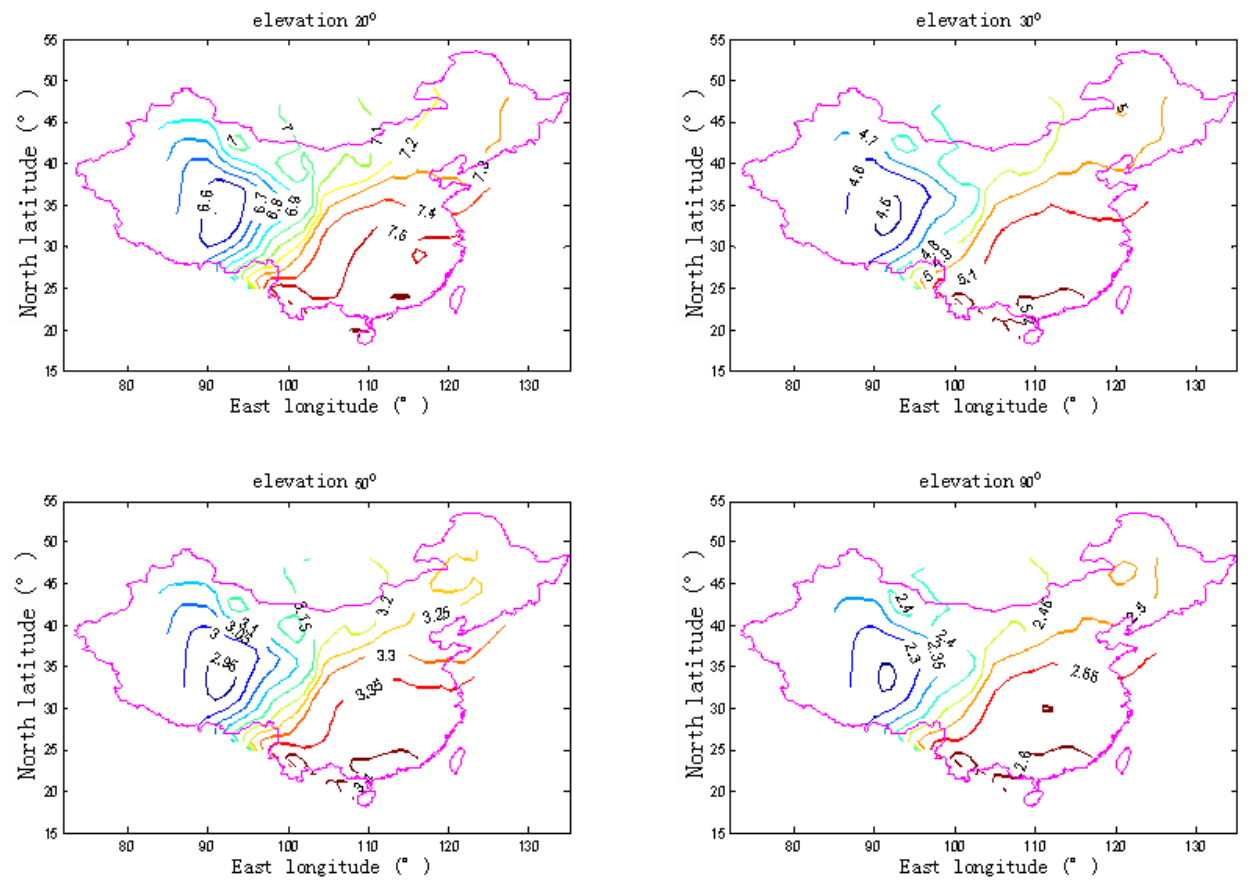

Distance refractive error in August (m)

Figure 3. The inland distribution of distance error caused by Tropospheric atmosphere refraction.

\subsection{Ionosphere Atmospheric Refraction Error}

The refractive error of Ionospheric atmosphere has a close relationship to systemic working frequency, degree of solar activity, etc. To take Haiko as an illustration, the changing of distance error caused by Ionosphere atmospheric refraction with solar activity and working frequency is shown in Figure 4, which is achieved by using the reference Ionosphere model.

By the analysis, we can get the following conclusion: when the frequency is in the $\mathrm{X}$ band and the elevation of the satellite is above $30^{\circ}$ (viewed from the ground), the maximal distance error caused by Ionosphere atmospheric refraction is about $0.5 \mathrm{~m}$, even in high solar activity years.

\section{Error Correction Technique for Atmospheric Refraction}

Distance errors caused by atmospheric refraction must be corrected to meet the accuracy demand of slant ranging in InSAR system with distributed satellites.

\subsection{The Overall Technical Process}

The overall technical process of distance error correction for atmospheric refraction is shown in Figure 5.

The key technoique mainly includes the following three aspects:

1) Methods of radio wave refraction correction.

2) Researches on profile model of global tropospheric refractive index. 


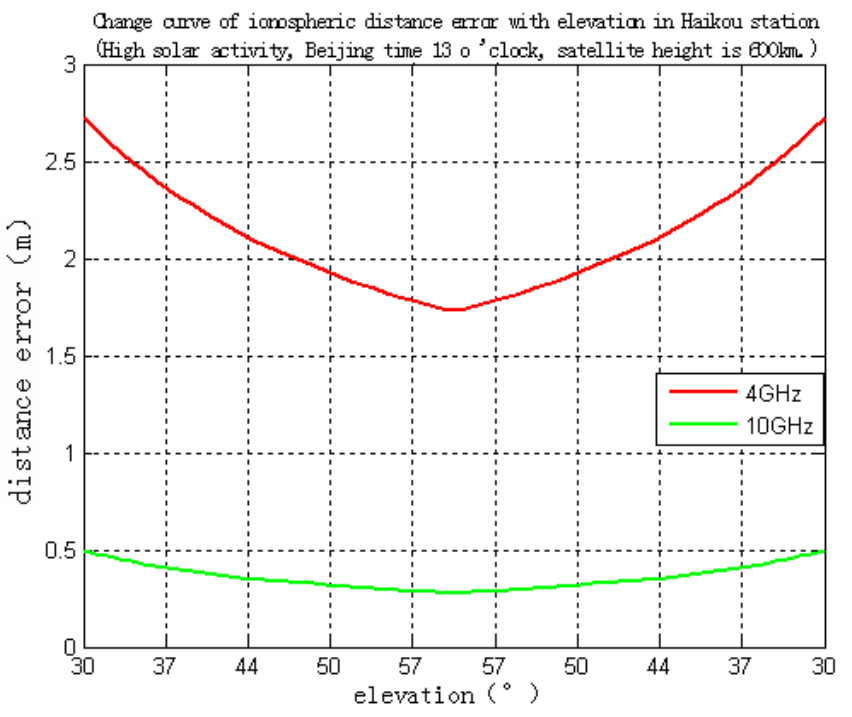

(a)

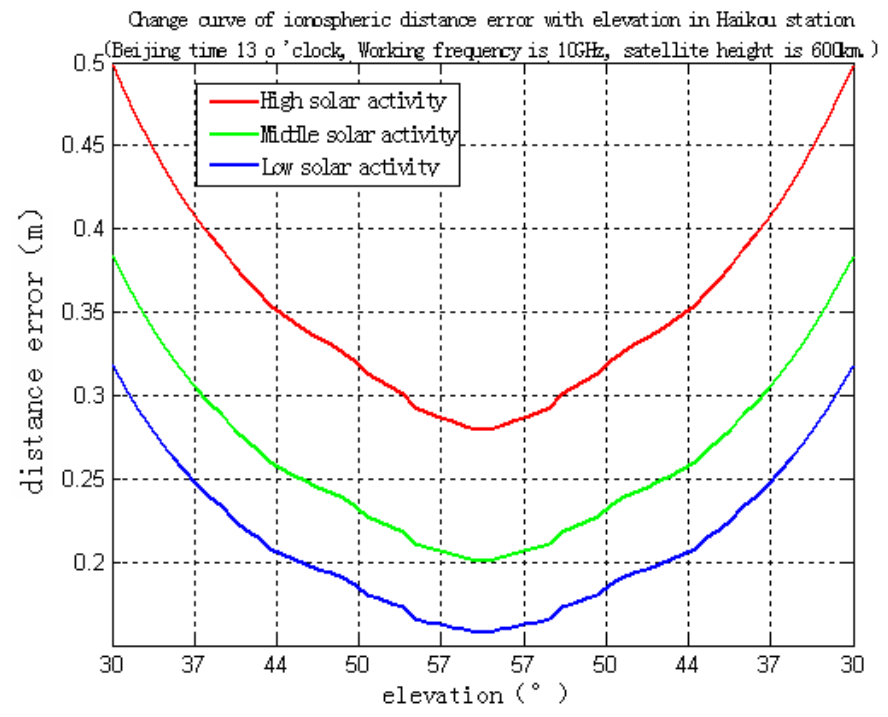

(b)

Figure 4. The change trend of the refraction error caused by Ionospheric atmosphere. (a) Change trend with frequency; (b) Change trend with solar activity.

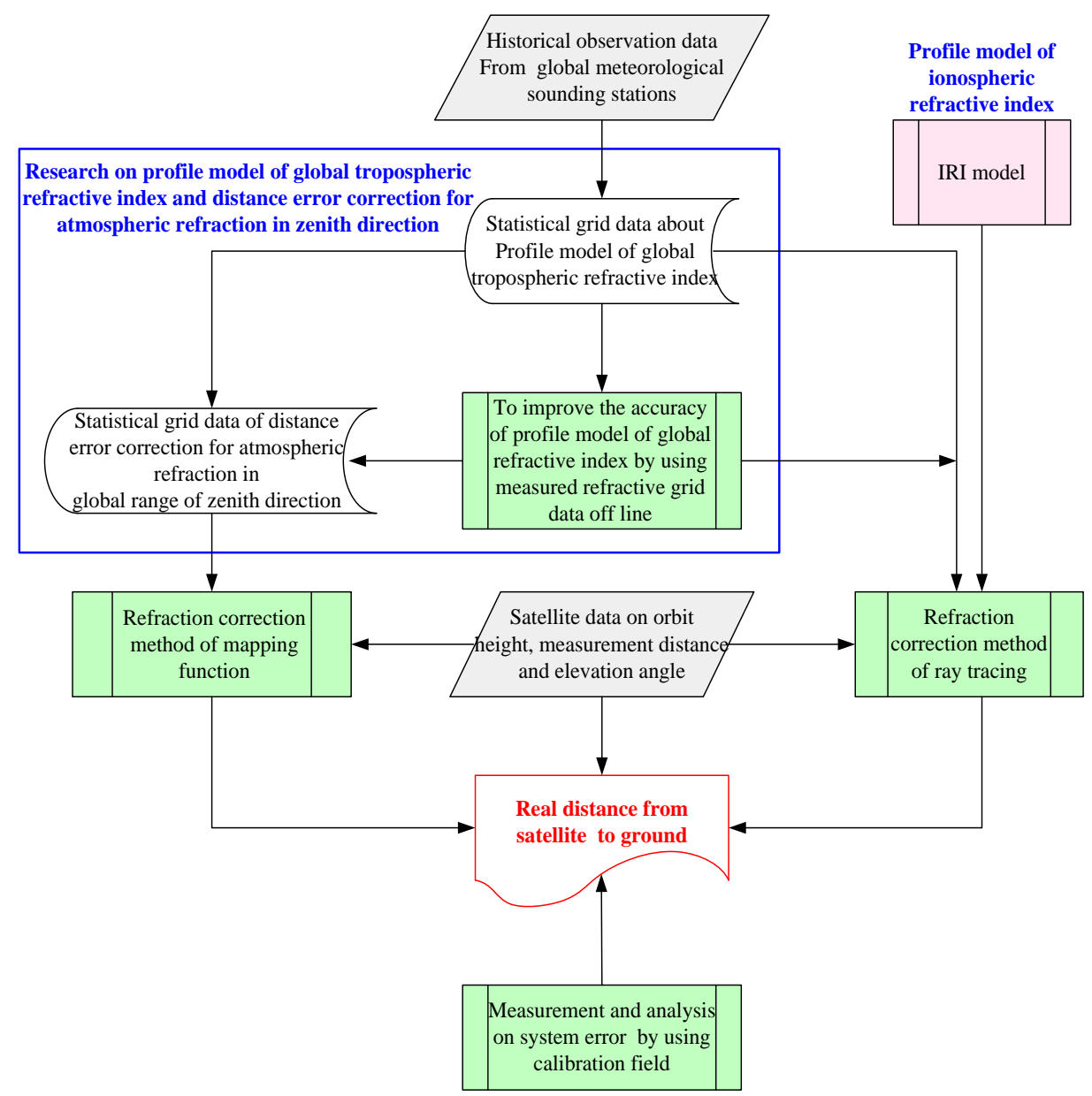

Figure 5. The overall technical process. 
3) Methods of system error calibration.

\subsection{Realization of Key Technique}

\section{1) Refraction correction method of mapping function}

In view of the high elevation of the system, we can use the refraction correction method of mapping function under the premise of the accuracy being not affected.

The method adopts a mapping function as the following form.

$$
\Delta L=\frac{\Delta L_{V}}{\sin \theta_{0}\left(1+k \cot ^{2} \theta_{0}\right)^{1 / 2}}+\delta\left(\theta_{0}, \Delta L_{V}\right)
$$

where $\Delta L_{V}$ is the distance error of refraction in zenith direction, $\theta_{0}$ is exterior angle, $k=1-\left(\frac{n_{0} r_{0}}{n(H) r(H)}\right)^{2}$, and the feature height $H=10^{6} \times \frac{\Delta L_{V}}{N_{0}}$.

\section{2) Profile model of global tropospheric refractive index}

To achieve the global distance error correction for refraction, it is necessary to establish the profile model of global tropospheric refractive index. Firstly, a global range of refractive index profile model of statistical data is established by using a great deal of historical meteorological sounding data obtained from the global observation stations. Secondly, the statistical model is corrected afterwards to improve the precision of the profile model by using the measured refractive index of global.

\section{3) System error calibration}

Microwave radiometer, which has a good real-time performance, can observe automatically and continuously on atmospheric environment in all-weather conditions. And it can directly calculate the radio wave refraction error of the propagation path by measuring the atmospheric radiation brightness temperature of the propagation path. It is shown in the study that the accuracy of refraction correction can reach $2 \mathrm{~cm}$ in the elevation of above $100^{\circ}$. Accordingly, the refractive error which calculated from the microwave radiometer measurement data can be considered as the total error caused by the radio environment.

The famous Marcor technique, which measures the additional time-delay integral of atmosphere on the path of radio wave propagation with ground-based microwave radiometer, gives distance error correction directly [15].

Due to the fact that the elevation of the satellite with distributed SAR is more than $30^{\circ}$, ray bending effects can be ignored. Therefore, the distance error caused by atmospheric refraction can be formulated as follow.

$$
\begin{aligned}
\Delta R & =10^{-6} \int_{0}^{R} N d S \\
& =10^{-6}\left[\int_{0}^{R} N_{d} d S+\int_{0}^{R} N_{w} d S\right] \\
& =\Delta R_{d}+\Delta R_{w}
\end{aligned}
$$

where $N_{d}$ is the dry item of atmospheric refractive index, $N_{w}$ is the wet item of atmospheric refractive index, $\Delta R_{d}$ is the distance refractive error caused by the dry item, and $\Delta R_{w}$ is the distance refractive error caused by the wet item. 


\section{Summary and Conclusions}

Atmospheric refraction is the main one among the errors which influence the accuracy of the InSAR system. Distance errors caused by atmospheric refraction must be corrected to meet the accuracy demand of slant ranging in InSAR system with distributed satellites. Aiming at two satellites, error correction experiments were carried out in 2007 and 2008 by using the error correction method presented in the paper. The experiments have demonstrated that residual error is little and steady after using the distance error correction for atmospheric refraction. It shows that the method discussed in the paper is effective, which can mainly eliminate the distance precision loss caused by Troposphere atmospheric refraction and Ionosphere atmospheric refraction.

Further researches and experiments should be done in future to get a better atmospheric refraction model and a better error correction method.

\section{References}

[1] Massonnet, D. and Feigl, K.L. (1995) Discrimination of Geophysical Phenomenain Satellite Radar Interferograms. Geophysical Research Letters, 22, 1537-1540. https://doi.org/10.1029/95GL00711

[2] You, X.Z., Qiao, X.J. and Wang, Q. (2002) Synthetic Aperture Radar Interferometric and Its Application. Journal of Geodesy and Geodynamics, 22, 109-116.

[3] Zebker, H.A., Rosen, P.A. and Hensley, S. (1997) Atmospheric Effects in Interferometric Synthetic Aperture Radar Surface Deformation and Topographic Maps. Journal of Geophysical Research, 102, 75470-75631. https://doi.org/10.1029/96JB03804

[4] Hanssen, R. (1988) Atmospheric Heterogeneities in ERS Tandem SAR Interferometry. Delft University Press, Delft.

[5] Hanssen, R.F. (2001) Radar Interferometry: Data Interpretation and Error Analysis. Kluwer Academic, Dordrecht, Boston. https://doi.org/10.1007/0-306-47633-9

[6] Goldstein, R.M. (1995) Atmospheric Limitations of Repeat-track Radar Interferometry. Geophysical Research Letters, 22, 2517-2120. https://doi.org/10.1029/95GL02475

[7] Lai, X. and You, X.Z. (1988) Satellite Geodesy. Seimology Press, Beijing. (In Chinese)

[8] Green, R.M. (1985) Spherical Astronomy. Cambridge University Press, Cambridge.

[9] Saastamoinen, J. (1972) Atmospheric Correction for the Troposphere and Stratosphere in Radioranging of Satellites, in the Use of Artificial Satellites for Geodesy. Geophys. Monogr. Ser. (edited by Henriksen, S.W., et al.), 15, 247-251.

[10] Documentation for the GAMIT GPS Analysis Software Release 10.0, GAMIT and SCRIPPS, 2002.

[11] Hugentobler, U., Schaer, S. and Fridez, P. (2001) Documentation of the Bernese GPS Software Version 4.2. Astronomical Institute University of Berne.

[12] Liu, J., Kuga, Y. and Xiaoqing, P. (2003) Ionospheric Effects on SAR Imaging: A Numerical Study. IEEE Trans on Geosci. Remote Sensing, 41, 939-947.

[13] Liu, J. (2003) Ionospheric Effects on Synthetic Aperture Radar Imaging. Ph. D. Dissertation, University of Washington.

[14] Quegan, S. and Lamont, J. (1986) Ionospheric and Tropospheric Effects on Synthetic Aperture Radar Performance. Remote Sensing, 1, 525-539. 
https://doi.org/10.1080/01431168608954707

[15] Sandwell, D.T. and Price, E.J. (1998) Phase Gradient Approach to Stacking Interferograms. Journal of Geophysical Research, 103, 301830-302041.

Submit or recommend next manuscript to SCIRP and we will provide best service for you:

Accepting pre-submission inquiries through Email, Facebook, LinkedIn, Twitter, etc. A wide selection of journals (inclusive of 9 subjects, more than 200 journals)

Providing 24-hour high-quality service

User-friendly online submission system

Fair and swift peer-review system

Efficient typesetting and proofreading procedure

Display of the result of downloads and visits, as well as the number of cited articles

Maximum dissemination of your research work

Submit your manuscript at: http://papersubmission.scirp.org/

Or contact jcc@scirp.org 